\title{
COLECTOR SOLAR CONSTRUIDO MEDIANTE TALADRADO POR FLUENCIA TÉRMICA
}

\author{
Víctor Heredia R. ${ }^{1} \quad$ Juan Tapia L. $^{1} \quad$ Camilo Flores C. $^{1} \quad$ Juan M. Godoy R. $^{1} \quad$ Jorge Vergara Díaz $^{1}$ \\ Recibido el 28 de septiembre de 2004, aceptado el 21 de abril de 2005
}

\begin{abstract}
RESUMEN
Se diseñó y construyó un sistema de calentamiento de agua residencial, mediante dos colectores solares y un estanque. En el sistema se utilizaron tubos de cobre perforados por taladrado por fluencia térmica (TFT), unidos con soldadura de plata. El sistema funciona por termosifón y se emplea como fluido de calentamiento una mezcla de etilenglicol-agua. El fluido de calentamiento pasa a través de un serpentín de cobre a un estanque de 200 litros. Diariamente se calientan 80 litros de agua a una temperatura máxima de $45^{\circ} \mathrm{C}$.
\end{abstract}

Palabras clave: Manufactura de cobre, taladrado no convencional, energía no convencional, calentamiento solar.

\section{ABSTRACT}

A heating equipment for residential water was built, using two solar collectors and one isolated reservoir. The system uses to copper tubes perforated by thermal flow drilling (TFT), brazing with silver solder. The thermal fluid moves due to the density change of the cold and hot water. It is a mixture of ethyleneglycol-water and the heat is exchanged in the copper serpentine installed in the reservoir of 200 liters. Daily it warms 80 liters of water to $45^{\circ} \mathrm{C}$.

Keywords: Copper manufacturing, non conventional drilling, non conventional energy, solar heating.

\section{INTRODUCCIÓN}

Un sistema solar doméstico fue patentado en USA en 1891. En 1909 en Los Ángeles comienza la venta de calentadores solares en que el colector y el depósito estaban separados y funcionaban por el principio del termosifón. La tecnología para fabricar colectores solares está muy difundida en la literatura [1], [2]. El proceso de unión de tubos delgados exige una gran habilidad del operador, debido a la pequeña área de contacto entre las paredes de ambos tubos. Normalmente esta unión no puede ser sometida a esfuerzos de deformación o torsión por el riesgo de rotura. Para evitar este efecto, la rejilla debe ser colocada en una estructura adicional rígida.

La rigidez de la unión soldada puede ser mejorada e inclusive ser utilizada como estructura, en el caso de colectores solares fabricados con el proceso de taladrado por fluencia térmica. Este proceso, sin arranque de viruta [3], forma un manguito o buje en la pared del tubo perforado, debido al material que fluye durante el proceso. Este manguito o buje que puede ser visto en la Fig. 1 aumenta el área de contacto de la unión soldada y mejora notablemente la rigidez de la estructura [4].

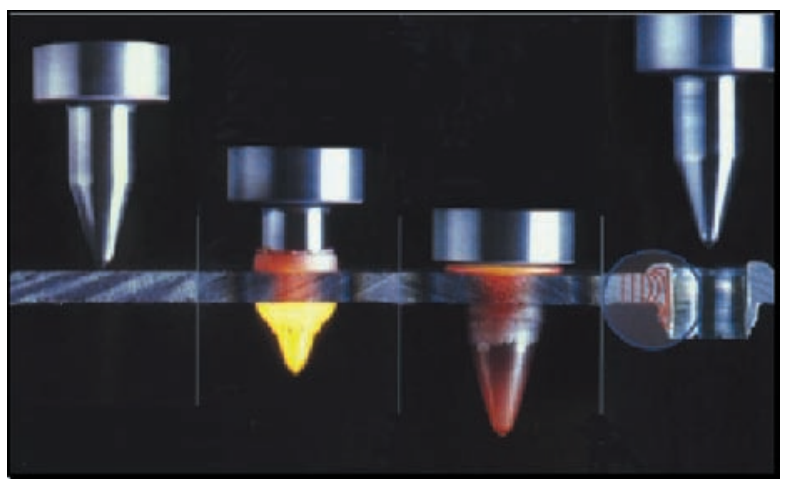

Fig. 1 Proceso de taladrado térmico por fluencia con herramienta de carburo de tungsteno.

1 Departamento Mecánica Universidad de Tarapacá, e-mail: jmgodoy@uta.cl, jvergara@uta.cl 


\section{PARTE EXPERIMENTAL}

\section{Diseño y fabricación de los colectores}

Se construyeron dos colectores solares de $2.000 \times 1.000$ $\times 100[\mathrm{~mm}]$.

La rejilla de cada colector está formada por nueve tubos de cobre de $12,8[\mathrm{~mm}]$ de diámetro externo $(3 / 8 ")$ y 1.840 $[\mathrm{mm}]$ de longitud, unidos en cada extremo a dos tubos de cobre de $25[\mathrm{~mm}]$ de diámetro (1") y $1.000[\mathrm{~mm}]$ de longitud. La perforación de los tubos de $25[\mathrm{~mm}]$ fue realizada mediante taladrado por fluencia térmica y la unión perpendicular con el tubo de $3 / 8$ " fue realizada con soldadura de plata.

El taladrado por fluencia térmica forma un manguito a medida que el material se desplaza de la perforación por un proceso de fluencia [5]. Este manguito puede

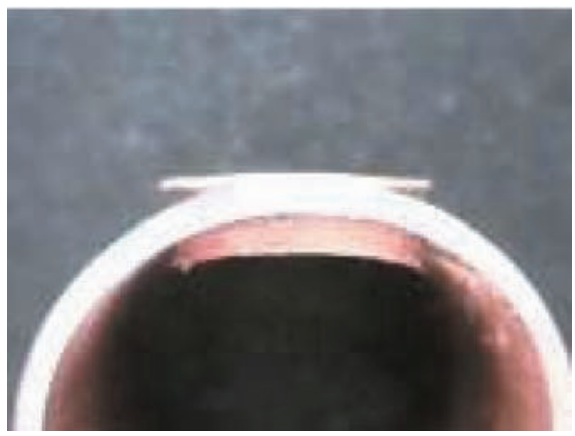

Fig. 2 Tubo de cobre de 1", velocidad $3.800 \mathrm{rpm}$, agujero guía $7 \mathrm{~mm}$.

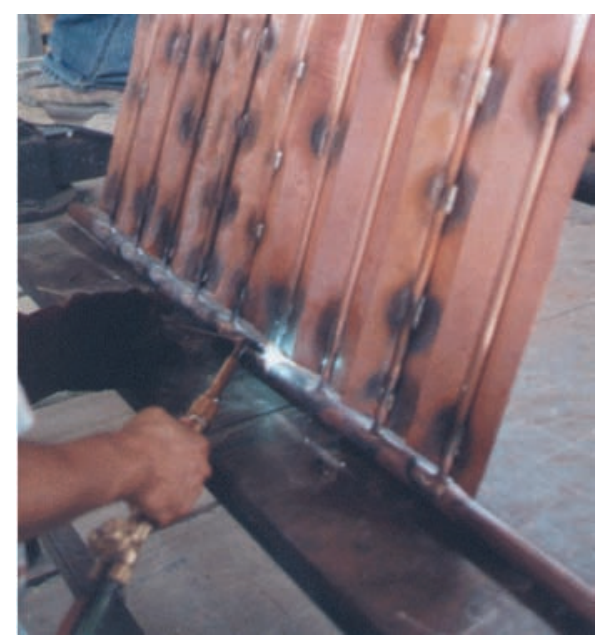

Fig. 3 Soldadura de la rejilla del colector solar. ser largo, corto, grueso o delgado de acuerdo al procedimiento utilizado [6]. La forma del buje se muestra en la Fig. 2.

Posteriormente se soldó a cada tubería de $3 / 8$ " una plancha de cobre de $0,3[\mathrm{~mm}]$ de espesor, de $100[\mathrm{~mm}]$ de ancho y $1.700[\mathrm{~mm}]$ de longitud para aumentar el área de absorción de calor. Estas planchas fueron soldadas con soldadura plomo estaño. Se observan estas planchas en las Figs. 3 y 4.

Finalmente la rejilla fue revestida con pintura negra e instalada en una estructura metálica junto con fibra aislante de $50[\mathrm{~mm}]$ de espesor. En la superficie se colocó un vidrio de $4[\mathrm{~mm}]$ de espesor, como puede ser visto en la Fig. 5.

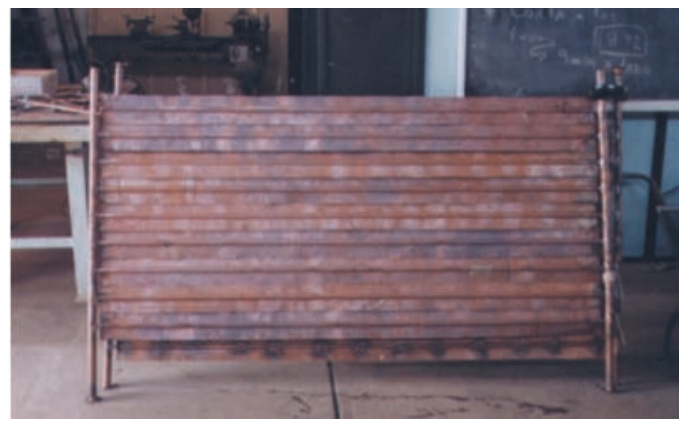

Fig. 4 Placa colectora terminada, antes de aplicar pintura negra.

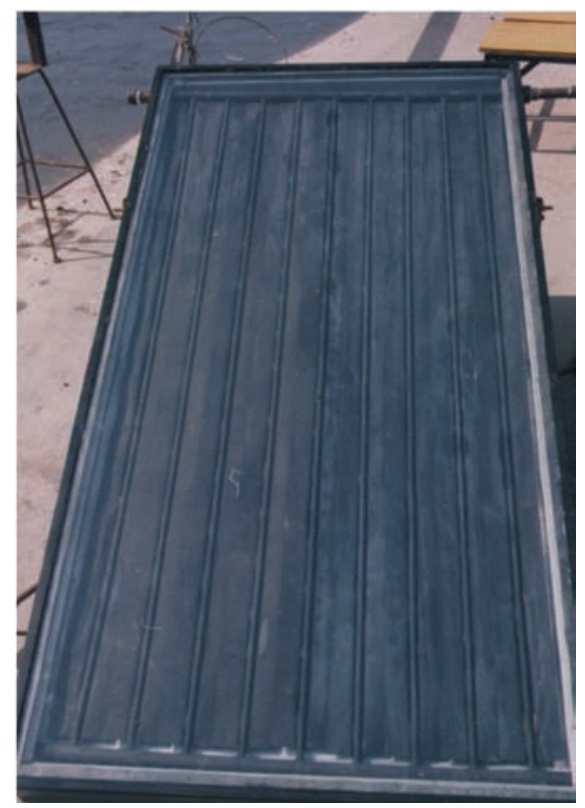

Fig. 5 Colector solar terminado, incluye aislante y vidrio. 


\section{Diseño y fabricación del estanque}

Para la fabricación del estanque se optó por un cilindro de acero de $4[\mathrm{~mm}]$ de espesor con un diámetro de 500 [mm] y una altura de $1.000[\mathrm{~mm}]$ y una capacidad de 200 litros. Este estanque fue revestido con lana de vidrio de $50[\mathrm{~mm}]$ de espesor. En la mitad inferior del estanque se instaló un serpentín con cañerías de cobre de 19 [mm] de diámetro (3/4") y de un largo de 14 [m], formando una espiral de 13 vueltas con un diámetro cercano a 340 $[\mathrm{mm}]$. Para determinar la longitud del serpentín se utilizó el valor de $0,25 \mathrm{~m}^{2}$ por metro cuadrado de colector [7]. El colector solar tiene $3,4 \mathrm{~m}^{2}$, lo que corresponde a un área de $0,85 \mathrm{~m}^{2}$. Se utilizó un tubo de cobre de 3/4", lo que da un largo de 14 metros.

Un esquema del estanque es mostrado en la Fig. 6. Este serpentín intercambia el calor entre el fluido etilenglicolagua calentado en los colectores, que lo traspasa al agua del estanque. El estanque tiene una entrada de agua potable en la parte superior mediante un sistema de flotador. La salida del agua es por la parte inferior y se controla con una válvula termostática.

Las conexiones entre los colectores y el estanque se realizaron con tuberías de cobre de 3/4" de diámetro, todas las líneas fueron revestidas con poliuretano de 50 $\mathrm{mm}$. Para llenar el circuito del fluido de calentamiento se instaló un depósito de expansión abierto, también revestido con poliuretano.

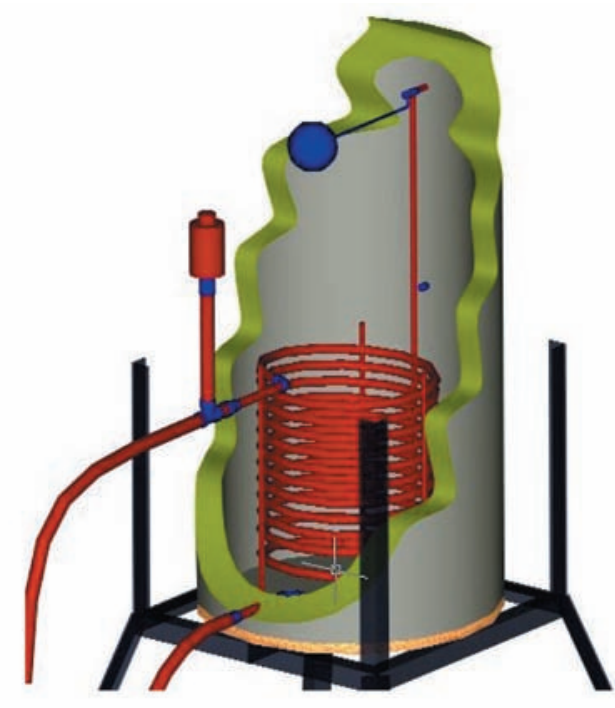

Fig. 6 Detalle interior del estanque de almacenamiento de agua caliente.

\section{Sistema de termosifón}

Debido al elevado contenido de sólidos del agua potable de Arica se utilizó un sistema de termosifón usando como fluido de intercambio etilenglicol-agua. Este sistema aprovecha la diferencia de temperatura del líquido en el colector y en el estanque, la que ocasiona una diferencia de peso específico, que a su vez produce el movimiento del líquido desde la zona caliente a la zona de menor temperatura en el estanque.

De este modo la posible incrustación de sales se presenta por la parte externa del serpentín, permitiendo así la limpieza por su parte externa, lo que disminuye el tiempo de limpieza. El circuito se muestra en la Fig. 7.

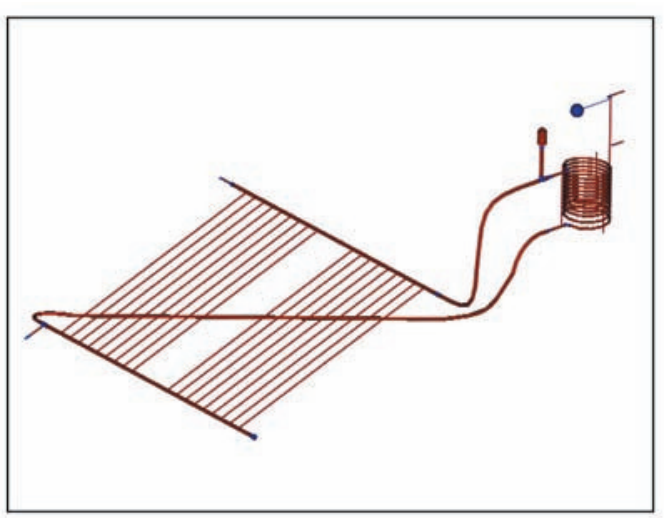

Fig. 7 Detalle del circuito primario de etilenglicol.

El sistema de paneles solares y estanque construido se muestra en la Fig. 8.

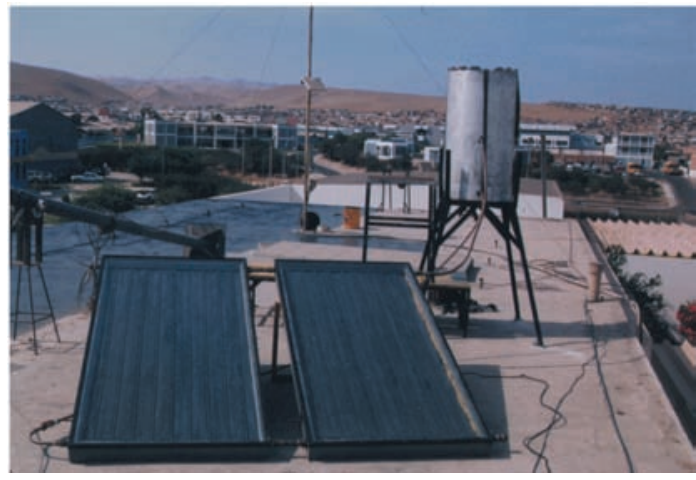

Fig. 8 Sistema paneles solares estanque, inclinación $19^{\circ}$ orientado hacia el Norte. 


\section{Rigidez de la rejilla. Soldadura}

Se observó microscópicamente que las soldaduras llenan completamente el espacio de contacto entre los tubos.

La resistencia a la soldadura se midió en la Máquina Universal de Ensayos, mediante un dispositivo diseñado y fabricado en el Departamento de Mecánica. El dispositivo puede ser visto en la Fig. 9.

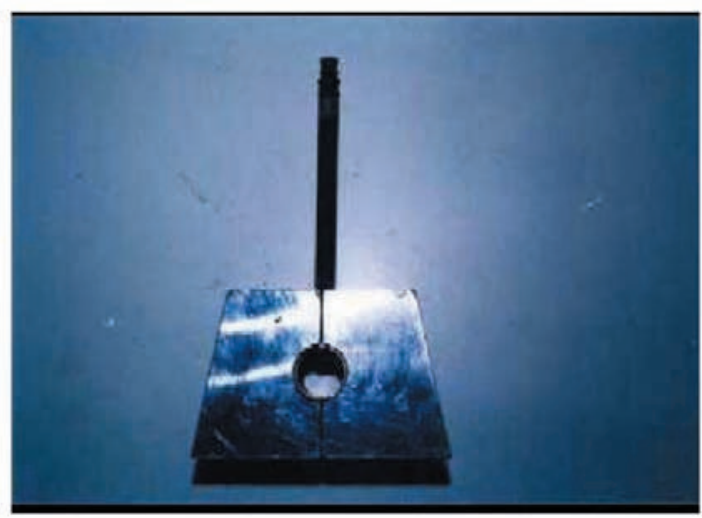

Fig. 9 Dispositivo para ensayar soldaduras en tubos de cobre.

Las uniones soldadas no se rompieron en la zona soldada. El rompimiento fue en el tubo de cobre de $3 / 8$ " adyacente a la zona soldada y no en la soldadura. En la Fig. 10 se muestra el rompimiento del tubo. El ensayo de tracción indicó una resistencia del tubo de $210 \mathrm{MPa}$, similar a la resistencia a la tracción del cobre recocido [8].

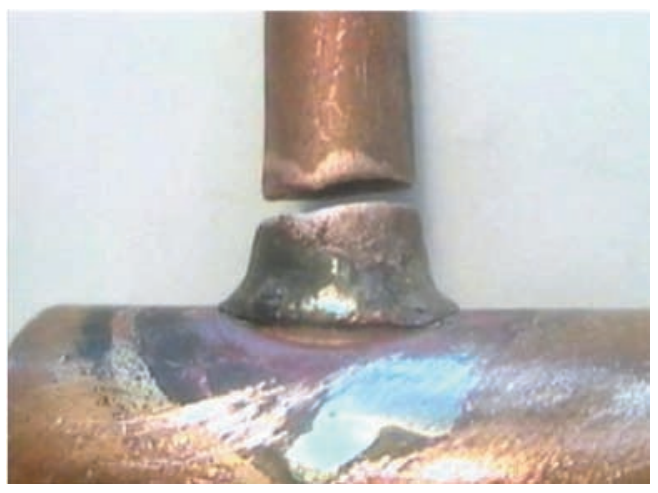

Fig. 10 Rotura del tubo de 3/8" en la Máquina Universal de Ensayos.

\section{RESULTADOS}

\section{Soldadura de la rejilla}

La rejilla fabricada mediante taladrado térmico por fluencia podrá funcionar como estructura en los próximos colectores solares reduciendo el peso y haciendo el panel más económico. Se ha fabricado un nuevo colector solar substituyendo la estructura metálica por plástico reforzado con fibra de vidrio.

\section{Rendimiento térmico}

Durante el mes de febrero del 2003 se midieron las temperaturas en la entrada y salida del agua en los colectores solares. La variación de la temperatura puede ser observada en la Fig. 11. Se verificó que la máxima temperatura se alcanza entre dos horas antes y dos horas después del medio día solar. Si el consumo es realizado en la noche, en la mañana no existirá agua disponible para el baño.

También se determinó la temperatura del agua en el estanque a $300[\mathrm{~mm}]$ desde la base, como puede ser observado en la Fig. 12. La disminución de la temperatura de $40^{\circ} \mathrm{C}$ hasta $29^{\circ} \mathrm{C}$ se debe a la retirada del agua del estanque para realizar un nuevo experimento.

El rendimiento térmico del sistema corresponde a la relación entre la energía útil y la radiación solar total que incide sobre el sistema. Como la energía útil depende de la temperatura del absorbedor (Te) y la temperatura ambiente (Ta), su valor dependerá de la radiación (I) y de la temperatura del agua en el absorbedor. Es decir, existirá un rendimiento instantáneo y un rendimiento mensual. El rendimiento alcanzado por el colector fue del $50 \%$, similar al rendimiento teórico indicado en la literatura para colectores solares en la región de AricaChile. La Ecuación es la siguiente [7]:

$$
\eta=a-\frac{b(T e-T a)}{I}
$$




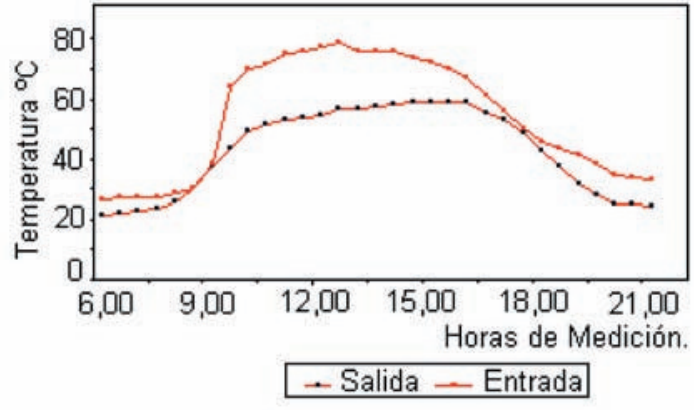

Fig. 11 Gráfico de temperaturas de entrada y salida de los colectores solares.

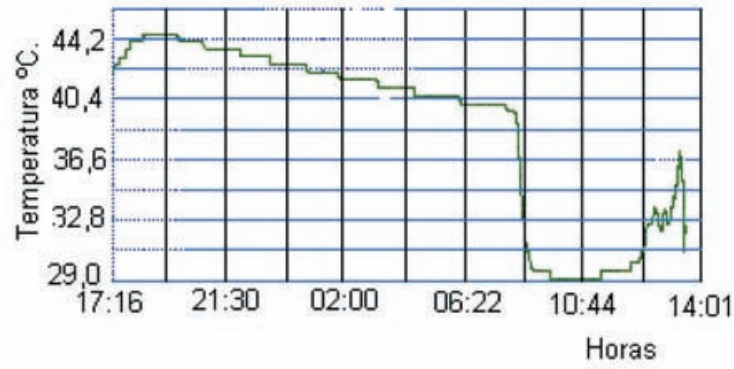

Fig. 12 Gráfico de la temperatura del agua en el estanque a $300[\mathrm{~mm}]$ de altura desde la base. Días 6 y 7 febrero 2003.

\section{Evaluación económica}

Una evaluación económica preliminar del sistema solar nos muestra un costo fijo de US $\$ 1.380$ y el calentamiento de 29.200 litros de agua desde 20 a $45^{\circ} \mathrm{C}$ en un año. El costo inicial del sistema equivalente de gas indica un costo fijo de US \$280 y para calentar la misma cantidad de agua un costo de operación anual de $\$ 170$. Se observa que después de 7 años, los costos son similares. La vida útil de un calefón es de 7 años, mientras que un colector solar puede ser usado durante 15 años, siempre que se realice una mantención preventiva adecuada.

Los costos de mantención no fueron incluidos en la evaluación ya que ambos sistemas necesitan este servicio. En el caso del colector solar se deben mantener los vidrios limpios para que la radiación se aproveche en su totalidad. Como el agua nacional tiene muchos iones, es necesario cada año hacer una revisión de la parte exterior del serpentín en el estanque de agua y si es necesario sacarlo y limpiarlo. Se debe evitar cualquier tipo de filtración que se pueda presentar con el uso. Con el serpentín se evita la incrustación en los tubos de los colectores solares, problema recurrente en la instalación de sistemas solares sin circuito adicional.

\section{CONCLUSIONES}

Se ha construido un sistema de calentamiento de agua residencial con colectores solares por taladrado por fluencia térmica. Mediante este proceso se pueden disminuir los costos de construcción de paneles solares.

Este sistema de calentamiento presenta rendimientos térmicos similares a los valores teóricos indicados en la literatura para la ciudad de Arica.

El colector solar es rentable después de 7 años, es un generador de energía limpia, no contamina la atmósfera y en la zona norte está disponible todo el año. El sistema a gas depende de un recurso importado, además contamina la atmósfera y su valor en un futuro cercano no se conoce.

\section{AGRADECIMIENTOS}

Se agradece a la Dirección de Investigación y Posgrado de la UTA, que financió este proyecto de investigación $\mathrm{N}^{\circ} 8741-01$

\section{REFERENCIAS}

[1] B02 Instalaciones de Equipos Solares Domésticos. Prosol. Norma Española. Junio 1999.

[2] A. Themmessl y W. Weiss. Solaranlagen Selbstbau. Erneuerbare Energie. Gleisdorf. Osterreich. 1999

[3] A.H. Streppel. "Flow drilling: a preliminary analysis of a new bus making operation". Annals of the CIRP. Vol. 32. 1983.

[4] W. Weingaerner, J.C. López da Silva, F. Tikal y R. Heiler. "Furação em chapas por escoamento: uma nova tecnologia de fabricação". Máquinas e metais. Sept. 1996, no. 368: 85-97. Brasil.

[5] J. Vergara, S. Damm, J. Villanueva, J.M. Godoy y F. Tikal. "Bush making by thermal flow drilling in copper and brass". The International Journal for Manufacturing Science \& Production, vol. 4, no. 2. 2001.

[6] J.M. Godoy, J. Vergara, J. Villanueva, M. Quispe, P. Oviedo, F. Tikal, S. Damm. "Cutting forces measurements with a piezoelectric dynamometer during thermal flow drilling". Revista de la 
Facultad de Ingeniería - Universidad de Tarapacá, jun. 2003, vol. 11, no. 1: 65- 69. ISSN 0718-1337.

[7] T. Cabirol, A. Pelissou y D. Roux. El Calentador Solar de Agua. Manuel Company Editores. Barcelona. 1984.

[8] R. Carmona, J. Vergara y J.M. Godoy. Estudio de la soldadura de uniones de cobre por taladrado térmico. $2^{\circ}$ Congreso Internacional de Metalurgia de Transformación. Cusco, Perú. Nov. 2003. 